MATEC Web of Conferences 32, 07001 (2015)

DOI: $10.1051 /$ matecconf/20153207001

(C) Owned by the authors, published by EDP Sciences, 2015

\title{
Deep Tissue Wavefront Estimation for Sensorless Aberration Correction
}

\author{
Emina Ibrahimovic, Xiaodong Tao ${ }^{a}$, Marc Reinig, Qinggele Li and Joel Kubby \\ W.M. Keck Center for Adaptive Optical Microscopy, Jack Baskin School of Engineering, University of California, Santa Cruz, CA 95064, \\ USA
}

\begin{abstract}
The multiple light scattering in biological tissues limits the measurement depth for traditional wavefront sensor. The attenuated ballistic light and the background noise caused by the diffuse light give low signal to noise ratio for wavefront measurement. To overcome this issue, we introduced a wavefront estimation method based on a ray tracing algorithm to overcome this issue. With the knowledge of the refractive index of the medium, the wavefront is estimated by calculating optical path length of rays from the target inside of the samples. This method can provide not only the information of spherical aberration from the refractive-index mismatch between the medium and biological sample but also other aberrations caused by the irregular interface between them. Simulations based on different configurations are demonstrated in this paper.
\end{abstract}

\section{Introduction}

As light passes through biological tissue, it can be absorbed, refracted and scattered, limiting the resolution and depth of optical imaging in biological tissues. Overcoming these challenges will benefit a wide range of applications from basic biological research to clinical investigations. Although scattering will exponentially reduce the intensity of ballistic light with the imaging increasing depth, correction of refractive aberration still benefits the imaging resolution and contrast $[1,2]$. Wavefront correction can dramatically reduce the surrounding lobes of the point spread function. The advantages of the large isoplanatic angle and fast correction speed make it suitable for live imaging.

To correct the refractive aberration, Adaptive Optics (AO) with different strategies has been applied in optical microscopes [3]. However the performance of those systems relies heavily on the intensity of the ballistic light from the samples. In biological tissues, the ballistic light will be attenuated exponentially with increasing depth because of scattering. When using Shack-Hartmann wavefront sensing, the scattering will not only limit the amount of photons delivered to the guide-star, but also increase the background noise. In a recent study, near IR guide star has been used for a two photon microscope to extend the wavefront measurement depth [2]. Interferometric focusing has been also applied to a guide star to increase the SNR of the wavefront sensor [1].

An alternative way to correct aberration in a thick biological tissue is to use wavefront sensorless method. Instead of measuring the wavefront directly, the optimal phase is retrieve by maximizing the detected signal from samples [4] or calculating from the optical model of the

\footnotetext{
${ }^{a}$ Corresponding author: taoxd@soe.ucsc.edu
}

mediums $[5,6,7]$. The latter one becomes more useful when pushing imaging depth limit. The optical model for spherical aberration induced by refractive index (RI) mismatch between the medium and samples has been used to correction aberration in skin tissue, mouse brain tissue and of $C$. elegans [5,6,7]. Here we introduced a more general model which can take into account of the irregular interface.

\section{Methods}

The concept of wavefront calculation based on ray tracing method is shown in Fig. 1. Let's suppose the mediums between the target and objective can be separated into multiple uniform layers. The RI of each layer is given or can be measured. The rays from the target inside the sample propagate to the objective through those layers. With the single layer, such as waster in case of water immersion objective, the light from the objective forms a perfect spherical wavefront and focuses at the point $\mathrm{O}_{0}$. From this point, a perfect reference sphere can be constructed with the radius of $R$, which is as shown in Fig. 1. Then the ideal optical path length (OPL) of each ray from $\mathrm{O}_{0}$ to the reference sphere through the single medium can be determined by

$$
O P L_{\text {ideal }}=n_{M} R
$$

where $\mathrm{n}_{M}$ is the RI of the top layer. The ray from the target is defined in a spherical coordinate as

$$
l(\theta, \phi)=\left[\begin{array}{c}
\cos \theta \sin \varphi \\
\sin \theta \sin \varphi \\
\cos \varphi
\end{array}\right]
$$


where $\theta$ and $\varphi$ is azimuth angle and zenith angle. Then the optical aberration is calculated as the optical path difference between the ideal OPL and the real OPL of ray between the target and reference sphere,

$$
\mathrm{OPD}(\theta, \varphi)=L_{i}-\mathrm{OPL}_{\text {ideal }}=\sum_{j=1}^{M} n_{j} l_{j}-\mathrm{OPL}_{\text {ideal }}
$$

where $L_{i}$ is the OPL of the ray. $n_{j}$ is the RI of $j^{\text {th }}$ layer. $l_{j}$ is the length of the ray in $j^{\text {th }}$ layer. The final wavefront in the pupil plane $W\left(r, \varphi^{\prime}\right)$ can be calculated from Eq. (2) by converting the spherical coordinate at the target point to the polar coordinate at the pupil plane. Suppose the ray $l(\theta, \varphi)$ from the target intersects the reference plane at $\mathrm{P}\left(\mathrm{p}_{\mathrm{x}} \mathrm{p}_{\mathrm{y}} \mathrm{p}_{\mathrm{z}}\right)$. Then the coordinat of ray in the pupil plane is given by

$$
\begin{aligned}
& r=\sqrt{p_{x}{ }^{2}+p_{y}{ }^{2}} \\
& \varphi^{\prime}=\operatorname{atan}\left(p_{x}, p_{y}\right)
\end{aligned}
$$

where $\mathrm{p}_{\mathrm{x}}$ and $\mathrm{p}_{\mathrm{y}}$ are $\mathrm{x}$ and $\mathrm{y}$ coordinates, respectively. If the reference sphere is $k^{\text {th }}$ surface, the intersection point $P_{k}$ is calculated as

with

$$
P_{k}=P_{k-1}+d l_{k}
$$

$$
d=\frac{-\left(l_{k} \cdot P_{k-1}\right)+\sqrt{\left(l_{k} \cdot P_{k-1}\right)^{2}-\left\|l_{k}\right\|^{2}\left(\left\|P_{k-1}\right\|^{2}-R^{2}\right)}}{l_{k}}
$$

To calculate the OPL of rays in each layer, the ray tracing algorithm is implemented to obtain the intersection point and the normal to the interface. The ray is described by the location of the ray origin and the direction vector. For the ray from the point $P_{k-1}$, any point on the $k^{\text {th }}$ ray can be defined as

$$
P=P_{k-1}+t l_{k}
$$

where $t$ is the distance between $P$ and $P_{k-1}$. For a flat surface, such as the surface of the coverslip, it can be defined as

$$
\left(P-d_{k}\right) \cdot N_{k}=0
$$

where $\mathrm{N}_{\mathrm{k}}$ and $\mathrm{d}_{\mathrm{k}}$ are the surface normal to the intersection point of the $\mathrm{k}^{\text {th }}$ surface and the optical axis. The intersection point $P_{k}$ is given by

$$
P_{k}=P_{k-1}+t l_{k}=P_{k-1}+\frac{\left(d_{k}-P_{k-1}\right) \cdot N_{k}}{l_{k} \cdot N_{k}} l_{k}
$$

For a more general quadric surface, the intersection point can be found by solving a quadratic equation [8]. At the interface between two layers with different RI, the refraction factor is calculated as [9]

$$
l_{j+1}=\eta_{j} l_{j}-\left(\eta_{j}\left(N_{j} \cdot l_{j}\right)-\sqrt{1-\eta_{j}^{2}\left(1-\left(N_{j} \bullet l_{j}\right)^{2}\right)}\right) N_{j}
$$

with

$$
\eta_{j}=n_{j} / n_{j+1}
$$

where $n_{j}$ is the refractive index of the $\mathrm{j}^{\text {th }}$ layer. To evaluate the influence of the specific aberrations, the wavefront estimation is fit by Zernike polynomials up to 22 mode using the Least Square method. The Zernike polynomials used in this paper are show in Table 1.

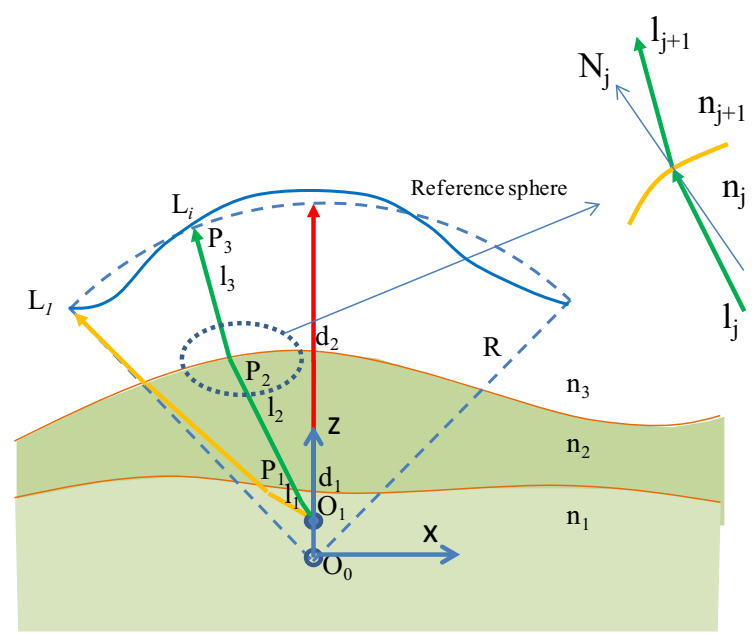

Figure 1. Wavefront calculation using the ray tracing method. $n_{1}, n_{2}$ and $n_{3}$ are the RI of the three layers. $\mathrm{O}_{0}$ is the ideal focusing point. $\mathrm{R}$ is the radius of the reference sphere. $l_{1}, l_{2}$ and $l_{3}$ are the rays in each layer. $d_{1}$ and $d_{2}$ are intersections between the optical axis and each layer. The ray intersection with each layer is at $P_{1}, P_{2}$ and $P_{3} . N_{j}$ is the normal to the surface.

\section{Simulation Results}

To demonstrate the wavefront estimation in optical microscopy using the ray tracing method, three medias are constructed in the program with RI of 1.35, 1.52 and 1.33 which corresponds to brain tissue, the cover glass and water. The thickness of the cover glass is set at $0.17 \mathrm{~mm}$. A water immersion objective with NA 1.05 is used in the simulation.

\subsection{Aberration induced from depth change}

We first demonstrate the aberration calculation at different depths. Since most commercial objectives can correct the aberration from the coverslip, we subtract all the wavefront estimation at different imaging depths with the one at the bottom of the coverslip. The wavefront at $1 \mathrm{~mm}$ depth in the tissue is shown in Fig. 2(a). The coefficient value of the first 22 Zernike polynomial modes is shown in Fig. 2(b). As can be seen, the major aberrations are spherical aberrations, which include both primary and secondary spherical aberration. Fig. 2(c) shows that the aberration changes linearly with the imaging depth, which agrees with previous research $[10,11]$ 


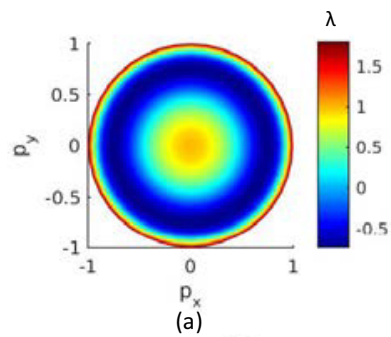

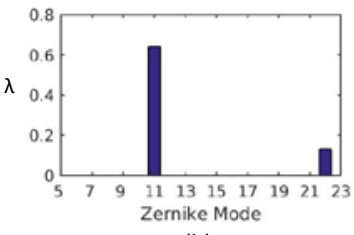

(b)

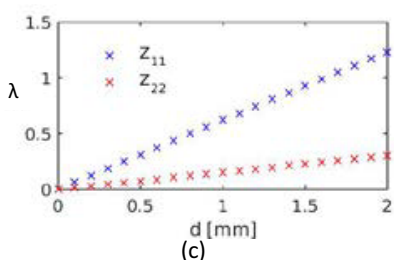

(c)
Figure 2. Aberration induced from depth change. (a) The Wavefront at the depth of $1 \mathrm{~mm}$ and (b) its first 22 Zernike coefficient values. (c) The spherical aberration changes with the depth. The color scale in (a) is keyed to wavelength

\subsection{Aberration induced from the coverslip tilt}

The water immersion objective is highly sensitive to the tilt of the coverslip. A titled window in a convergent beam generates coma and astigmatism aberrations. Using the correction collar on the objective lens, it is not possible to correct these aberrations. In this simulation, the cover slit is titled along $\mathrm{x}$ axis with a different angle. Fig. 3(a) shows the wavefront with a tilt angle of 1 degree. Fig. 3 (b) shows that the major aberrations are coma, astigmatism and Trefoil $\mathrm{x}$, where Coma $\mathrm{x}\left(\mathrm{Z}_{7}\right)$ gives the greatest contribution. Fig. 3(c) shows the nonlinear relationship between the tilt angle and the six main aberrations.

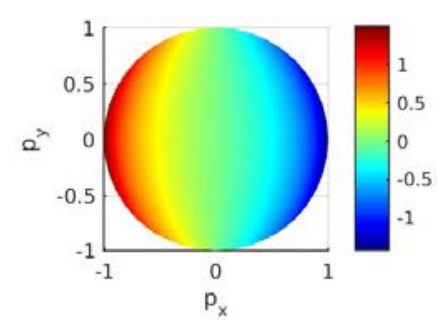

(a)

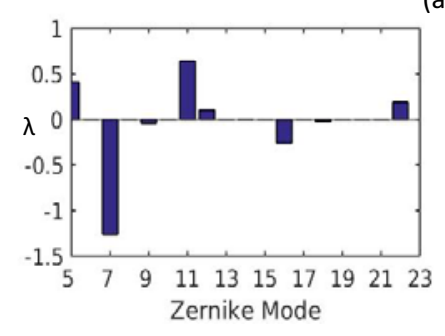

(b)

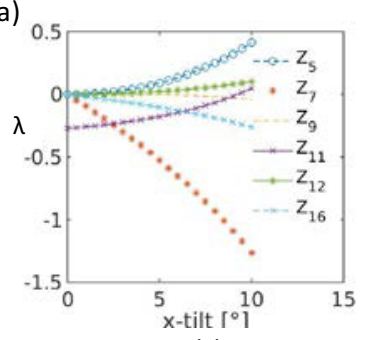

(c)
Figure 3. Aberration induced from the coverslip tilt. (a) The Wavefront at the tilt angle of 1 degree and (b) its first 22 Zernike coefficient value. (c) The aberrations change with the depth. The color scale in (a) is keyed to wavelength.
Table 1. Zernike polynomials used in this paper

\begin{tabular}{|c|c|c|}
\hline $\begin{array}{c}\text { Index } \\
(\mathrm{j})\end{array}$ & $Z_{n}^{m}(\rho, \theta)$ & Aberration mode \\
\hline 1 & 1 & Piston \\
\hline 2 & $2 r \cos (\theta)$ & Tilt $\mathrm{x}$ \\
\hline 3 & $2 r \sin (\theta)$ & Tilt $\mathrm{y}$ \\
\hline 4 & $\sqrt{3}\left(2 r^{2}-1\right)$ & Defocus \\
\hline 5 & $\sqrt{6} r^{2} \cos (2 \theta)$ & Astigmatism $\mathrm{x}$ \\
\hline 7 & $2 \sqrt{2}\left(3 r^{3}-2 r\right) \cos (\theta)$ & Coma $\mathrm{x}$ \\
\hline 9 & $2 \sqrt{2} r^{3} \cos (3 \theta)$ & Trefoil $\mathrm{x}$ \\
\hline 11 & $\sqrt{5}\left(6 r^{4}-6 r^{2}+1\right)$ & Primary Spherical \\
\hline 12 & $\sqrt{10}\left(4 r^{4}-3 r^{2}\right) \cos (2 \theta)$ & $\begin{array}{c}\text { Secondary } \\
\text { Astigmatism } \mathrm{x}\end{array}$ \\
\hline 16 & $\sqrt{12}\left(10 r^{5}-12 r^{3}+3 r^{2}\right) \cos (\theta)$ & Secondary Coma $\mathrm{x}$ \\
\hline 22 & $\sqrt{7}\left(20 r^{6}-30 r^{4}+12 r^{2}-1\right)$ & Secondary Spherical \\
\hline
\end{tabular}

\section{Conclusions}

In summary, this paper demonstrates a wavefront estimation method using a ray tracing algorithm. The wavefront is calculated by the OPL difference between a reference sphere and the real rays through different media. This method has potential to calculate the wavefront from more complex interfaces. The simulation shows the aberrations change with the image depth and tilt angle of the cover slit. The simulation results will be tested in a future experiment.

\section{Acknowledgments}

The results presented herein were obtained at the W.M. Keck Center for Adaptive Optical Microscopy (CfAOM) at University of California Santa Cruz. The CfAOM was made possible by the generous financial support of the W.M. Keck Foundation. This material is based upon work supported by the National Science Foundation under Grant Numbers 1353461 and 1429810. Any opinions, findings, and conclusions or recommendations expressed in this material are those of the authors and do not necessarily reflect the views of the National Science Foundation.

\section{References}

1. K. Wang, W. Sun, C. T. Richie, B. K. Harvey, E. Betzig, N. Ji, Nature Communications 6, 7276 (2015).

2. X. Tao, Z. Dean, C. Chien, O. Azucena, D. Bodington, J. Kubby, Optics Express 21, 3128231292 (2013).

3. J. A. Kubby, Adaptive Optics for Biological Imaging, (CRC Press, 2013).

4. D. Débarre, E. J. Botcherby, T. Watanabe, S. Srinivas, M. J. Booth, and T. Wilson Opt. Lett. 34, 2495-2497 (2009). 
5. N. Matsumoto, T. Inoue, A. Matsumoto, S. Okazaki, Biomed. Opt. Express 6, 2575-2587 (2015).

6. M. J. Booth, T. Wilson, J. Microsc. 200, 68-74 (2000).

7. P. Kner, J. W. Sedat, D. A. Agard, and Z. Kam, J. Microsc.237(2), 136-147 (2010).

8. A. S. Glassner An Introduction to Ray Tracing, (Academic Press, 1989)

9. J. D. Foley, A. van Dam, S. K. Feimer, J. F. Hughes, Computer Graphics, Principles and Practice. (Addison-Wesley, 1990).

10. S. Hell, G. Reiner, C. Cremer, E. Stelzer, J. Microsc. 169 (3), 391, (1993).

11. M. Booth, M. Neil, T. Wilson, J. Microsc. 192 (2), 90 (1998). 